\title{
A Novel Image Based Verification Method for Respiratory Motion Management in Radiation Therapy
}

\author{
Ali Khamene ${ }^{1}$, Christian Schaller ${ }^{2}$, Mamadou Diallo ${ }^{1}$, Juan Carlos Celi ${ }^{3}$, \\ Barbara Ofstad ${ }^{3}$, Joachim Hornegger ${ }^{2}$, X. Allen $\mathrm{Li}^{4}$, An Tai ${ }^{4}$, and John \\ Bayouth $^{5}$ \\ 1 Imaging and Visualization Dept., Siemens Corporate Research, 755 College Road \\ East, Princeton NJ 08540, USA \\ 2 Lehrstuhl fuer Mustererkennung, University of Erlangen-Nuremberg, Marten Str. 3, \\ 91058 Erlangen Germany \\ 3 Siemens Oncology Care Systems, Hans Bunte Str. 10, 69123 Heidelberg, Germany \\ 4 Medical College of Wisconsin, Department of Radiation Oncology, 8701 Watertown \\ Plan Road, Milwaukee WI 53226, USA \\ ${ }^{5}$ University of Iowa, Dept. of Radiation Oncology, 200 Hawkins Dr., LL West \\ Addition, Iowa City IA 52242, USA \\ ali.khamene@siemens.com
}

\begin{abstract}
Precise localization of moving targets in the thorax and abdomen during the course of treatment can be used to increase local control via dose escalation while reducing the severity of normal tissue complication. Localization of targets in real time with radio-opaque marker is not practical considering the excess radiation dose to the patient and potential complications of implantation. Various external surrogates can be used to give indications of locations of the targets during the breathing process. However, there is a great deal of uncertainty in correlation between external surrogates and internal target positions/trajectory during respiratory cycles. This is due to the fact that the correlation tends to vary through time as the patient's breathing pattern changes. In this paper, we address the problem of establishing the correlation between a surrogate signal and an internal target prior to treatment. We have developed an algorithm that automatically establishes correspondences between the fluoroscopic sequence frames taken from the patient on the day of treatment and the various phases of a $4 \mathrm{DCT}$ planning data set. Image based mapping/synchronization procedure is performed using an underlying Markov model established for the breathing process. The mapping procedure is formulated as an optimization process and is solved efficiently using a dynamic programming technique. Results on the phantom, synthetic, and real patient data demonstrate the effectiveness of the proposed method in coping with respiratory correlation variations. The approach could primarily be used for automatic gating interval adaptation in the gated radiotherapy.
\end{abstract}




\section{Introduction and Background}

Radiation therapy is a treatment modality with primary goal of precisely delivering the prescribed dose to the tumor while minimizing the dose to surrounding healthy tissues and critical structures. Recent technological advances in radiation therapy enable delivering of a highly conformal radiation dose distribution to a morphologically complex target volume. Main sources of delivery errors include the inter- and intra-fraction target and organ motions.

\subsection{Respiratory Motion in Radiotherapy}

Intra-fraction organ motion is mainly caused by patient respiration. Respiration induced tumor motion has been studied indirectly by movements of organs, radio-opaque markers (implanted at the vicinity of the tumor) [12], and surrogate organ/structure [6]. Real-time imaging technologies can be used to observe either tumor motion or indirectly perceive the tumor motion [10]. It has been shown that the tumor motion can be as large as 2-6 cm [7]. The variation of the motion size as well as the breathing pattern makes the prediction of the tumor location very difficult.

There are three major approaches for dealing with tumor motion in radiation treatment. In the first method, which is clinically used, the patient is asked to hold the breath or do a forced shallow breathing. The drawback here is that the method requires patient compliance. Furthermore, the implementation of the strategy is rather subjective and it depends on the skill of the therapist and participation of the patient. In the second and third method, the patient is required to do free breathing and no compliance is expected. In the second method, which is usually referred to as "gating", the radiation beam is gated in a way that the tumor is only treated at the "prescribed or planned" position [8]. Whereas in the third method, which usually referred to as "tracking", the radiation beam or table is moved so that the tumor always remains at a fixed point within the treatment field [11]. Although, tracking has the advantage of a higher duty cycle and a shorter delivery time, it is technically challenging to implement [13]. In contrast, respiratory gating is more practical, and has been adopted in clinic practice by a number of cancer centers [7].

\section{$1.2 \quad$ Gated Radiotherapy}

We focus on the external gating approach that it is non-invasive and the external surrogate signals could be generated from strain gauges around the patient's thorax, optical markers on the patient skin, or airbag systems measuring air flow or temperature. On the other hand, having a real time external signal is not equivalent to knowing the location of the tumor at all times. Exploiting external signal blindly to turn the radiation beam on and off can cause significant errors. In particular, the correlation between the tumor motion and the surrogate signal may change from the planning phase to the treatment sessions. The major drawback of this method is the variability of the correlation between the external signal amplitude (marker position) and the internal target position. 
The main challenge of the gated treatment is to adapt the gating intervals based on up-to-date breathing pattern of the patient. This paper focuses on an image based method, which maps the up-to-date images of the patient to the various phases captured during the planning phase. The mapping function is then used to determine optimal gating interval for the breathing surrogate for a specific treatment session.

\section{Image Based Verification Technique for Gated Radiotherapy}

At the planning phase, in order to have a better understanding of the tumor motion, time resolved volumetric CT images (so-called 4DCT) are acquired (see figures 1a). A surrogate signal is usually used to resolve the projections prior to reconstruction into a set of phases (usually 8-12) [14]. After reconstruction, various phases of the $4 \mathrm{DCT}$ are represented by either an amplitude or phase from the surrogate signal. The 4DCT scans are processed and the gross target volume (GTV) of the tumor for each phase is determined. Furthermore, based on the tumor location, residual motion, and duty cycle, a gating phase interval that is delimited by two phases of the 4DCT is selected. The union of the GTVs from the phases within the gating interval forms a new volume that is usually referred to as internal target volume (ITV). Based on the prescribed dose and shape of the ITV, a treatment plan is devised. Furthermore, amplitude or phase of the surrogate signal at the two end phases of the gating phase interval signifies the planned gating surrogate interval.

At the day of treatment, the initial patient setup is performed. . In order to reduce the positioning bias due to respiratory motion, we select a single phase out of $4 \mathrm{DCT}$ that roughly resembles the same breathing phase apparent from the acquired 2D setup x-ray or portal images. An image guided method is then used to correct for the residual positioning error [5]. After the setup verification process, the patient is at the optimal treatment position. Since the surrogates signal correlation with the target position varies day by day, the gating intervals set during the planning phase might not be valid anymore. It is desirable to check and possibly to adapt the gating intervals using an up-to-date respiratory pattern seen in a pre-treatment fluoroscopic acquisition.

\subsection{Problem Statement}

The image based verification process starts with acquisition of a pre-treatment image sequence. Mega voltage or kilo voltage fluoroscopic acquisition synchronized with a breathing surrogate is considered (see figures $1 \mathrm{~b}$ ). We assume that the pre-treatment image sequence $\left\{I_{p t}(j)\right\}_{j \in[0, K-1]}$ has $K$ frames taken at intervals of $\delta t$ covering at least several respiratory cycles (both $j$ and $K$ are integers). Furthermore, a corresponding synchronized surrogate signal $\left\{s_{p t}(j)\right\}_{j \in[0, K-1]}$ is acquired. In both notations, superscript $p t$ stands for "pre-treatment". We also assume that we have a planning 4DCT acquisition, which includes $L$ phases of breathing. We generate a set of Digitally Reconstructed Radiographs (DRRs), $\left\{I_{p}(i)\right\}_{i \in[0, L-1]}$, using each phase of the planning 4 DCT (e.g., $i$ th) with the exact 


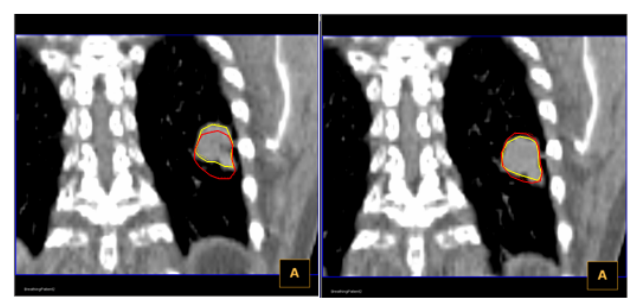

(a)

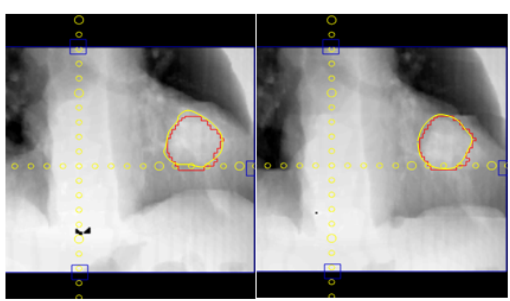

(b)

Fig. 1. (a) depicts the coronal slices of planning 4DCT data sets with visible contour lines. (b) demonstrates two frames of a fluoroscopic acquisition overlaid with the outlines of the projected structures from the planning $4 \mathrm{D}$ dataset.

geometry of the pre-treatment imaging system and the known patient position from the initial patient setup step (both $i$ and $L$ are integers). As mentioned in the previous section, the gating phase interval is delimited by two phases $i^{\text {min }}$ and $i^{\max }$ both within $[0, L-1]$. In order to map the gating phase interval, which is set at the planning step onto the surrogate signal acquired prior to treatment, we need to establish a mapping function $\mathcal{L}:[0, K-1] \mapsto[0, L]$ :

$$
\mathcal{L}(j)=\left\{\begin{array}{lr}
i \in[0, L-1] & \text { there is a correspondence for the frame } j \\
L & \text { no correspondence }
\end{array}\right.
$$

The mapping function needs to determined based on the image information from the two time series of $\left\{I_{p}(i)\right\}_{i \in[0, L-1]}$ and $\left\{I_{p t}(j)\right\}_{j \in[0, K-1]}$. Once the mapping function is estimated, the treatment day gating surrogate interval can be estimated as follows:

$$
s_{p t}^{\min }=\min _{j \mid \mathcal{L}(j) \in\left[i_{\min }, i_{\max }\right]} s_{p t}(j)
$$

For computing $s_{p t}^{\max }$, we switch the min to max in equation 2 . The treatment day gating surrogate interval $\left[s_{p t}^{\min }, s_{p t}^{\max }\right]$ can also be used to estimate a new duty cycle. The surrogate signal amplitudes of $s_{p t}(j)$ for $j$ satisfying the condition $\mathcal{L}(j) \in\left[i_{\text {min }}, i_{\text {max }}\right]$ can also be further analyzed to detect the outliers, mainly to increase the duty cycle as much as possible.

\subsection{Image Similarity Matrix}

The first step in establishing an optimal mapping function is to generate a similarity image matrix between the two image sequences of $\left\{I_{p}(i)\right\}_{i \in[0, L-1]}$ and $\left\{I_{p t}(j)\right\}_{j \in[0, K-1]}$. We generate a matrix $\mathbf{M}$ with size of $(L+1) \times K$, where $\mathbf{M}(i, j)$ (for $i<L)$ is the normalized mutual information value between the two images of $I_{p}(i)$ and $I_{p t}(i)$. Each column of the matrix $\mathbf{M}$ can be thought of as matching likelihood profile between a frame of the fluoroscopic sequence and 
various phases of the planning DRRs. A clear peak in that profile could signal a potential match. However, in the case where there is no match, one would expect to have consistently low value across the whole column. In order to deal properly with these cases, we have considered the last column, which is in fact representing the likelihood of having a "no-match" situation. For each element in the last row of the matrix $\mathbf{M}$, we consider a constant value that is the median of the maximums for various columns minus the range of the similarity values of the corresponding column.

Finding maximum values for each column of the matrix $\mathbf{M}$ could be a potential solution for the mapping function $\mathcal{L}$. However, the problem with this approach is that since each columns are processed independently, no temporal consistency is considered. For example, images from certain phases of inhale and exhale could look quite similar if they are observed independently and not considered in the context of a sequence. Not considering temporal aspect of the process also makes it difficult to deal with cases, where there is no match. Furthermore, robustness to noise and/or to variations of image intensities from the two sequences could be easily compromised.

\subsection{Respiratory Motion as A Markov Process}

In order to enforce temporal consistency in finding the mapping function $\mathcal{L}$ from the image similarity matrix $\mathbf{M}$, we first need to find a reasonable model for the breathing process [4]. We assume that the breathing can be modeled as a Markov process. We consider $L+1$ states for the Markov process; $L$ of which is based on the number of phases in the planning $4 \mathrm{DCT}$ and an additional one that accommodates variations beyond the observed states in the planning 4DCT. Since the pre-treatment acquisition is not triggered, it can start at any breathing phase, therefore the initial probabilities of the various states are constant(i.e. $P_{0}(i)=\frac{1}{L+2}$ for $\left.i \in[0, L+1]\right)$. We consider that the observations are identical to the process states, therefore the emission probability matrix is the identity. The state transition probability matrix $\mathbf{S}$, which is of size $(L+1) \times(L+1)$ can be estimated from a set of observation sequences using Baum-Welch algorithm [1]. Observation sequences, in this case, could be derived from various surrogate signals, where the signal amplitude is separated into $L+1$ bins.

We denote the states of the process as $S_{i}$ for $i \in[0, L]$, where $S_{L}$ is the state not present in the prior observations. $S_{0}$ denotes the start of inhale and $S_{L-1}$ indicates the end of exhale. The elements of the transition probability matrix $\mathbf{S}$ are $\mathbf{S}(i, j)=P\left(s_{n+1}=S_{j} \mid s_{n}=S_{i}\right)$ for $i, j \in[0, L]$, where $s_{n}$ indicates the state at the the time index $n$. Therefore, the sum of each column or row of the matrix $\mathbf{S}$ is one. Aside from being able to estimate the transition matrix given a series of sequences, there are many heuristic constraints that one might consider in determining the elements of the matrix $S_{i}$. For example, it is more likely to have an arrangement of states, where the state at $k+1$ has a higher state index than the state at $k$. The only exception is for $S_{L-1}$ that most likely transits back to state $S_{0}$. Furthermore, there is a possibility that the states repeat themselves. This case covers scenarios, where the breathing process is 


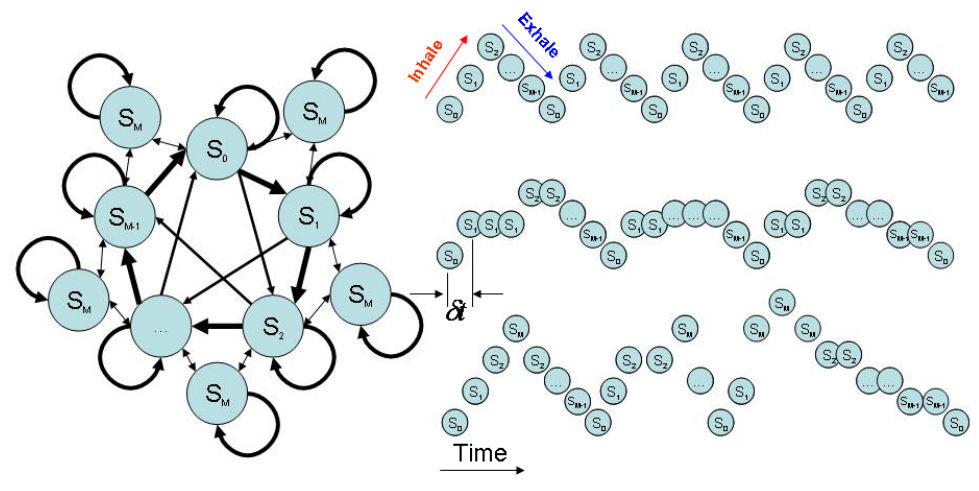

(a)

(b)

Fig. 2. (a) depicts the state transition network (thicker lines denote higher transition probability). (b) shows various instances of the sequences from the Markov process depicted at (a). Top most shows a most probable case. Middle row shows three breathing cycles, where the inhale is elongated in the first cycle, shallow breathing is present in the second cycle, and the slow breathing is shown in the last cycle. The bottom row depicts deep breathing in the first cycle, fast exhale and deep inhale in the second cycle, and fast and deep inhale and slow exhale in the last cycle.

slow. Furthermore, there should be a possibility for having forward transitions more than one step covering fast breathing scenarios. It is also reasonable to consider that the unknown state of $S_{L}$ is more likely to happen after the states closer to the full inhale or full exhale. Considering all these constraints, we consider the $L \times L$ sub-matrix from $\mathbf{S}$ to be a circulant matrix. The first column of the matrix has a negative quadratic form (i.e., maximum at the first element and quadratically decreasing as the index increases). The last row of the matrix represents the probability of the transition to an unknown state. For the last row, we consider to have a four degree polynomial, which maximizes at the mid range of the index (i.e., full inhale) and minimizes at the mid range of inhale and exhale. Finally, a normalization factor is applied to ensure that the sum of each column is one. The probability of a given sequence having been produced by a specific Markov process can be computed using the initial state probability $P_{0}$ and the transition matrix $\mathbf{S}$ using a forward-backward propagation algorithm [9]. An example of a heuristically determined transition matrix for the breathing cycle is depicted in figure 2 .

\subsection{Mapping as a Solution of an Optimization Problem}

The mapping function $\mathcal{L}$ is in fact a constellation of states $S_{i}$ for $i \in[0, L]$ from the planning phase. The likelihood of a certain mapping due to the imaging cues can be computed using the image similarity matrix M. Furthermore, the probability of a certain mapping solely due to the a priori Markov process model can be computed using $P_{0}$ and $\mathbf{S}$. Therefore, we setup an optimization to maximize 
the posteriori probability that is expanded to the product of the likelihood and prior probability using bayesian rule. The optimization formulation can then be written as:

$$
\widetilde{\mathcal{L}}=\arg \max _{\mathcal{L}}\left(\prod_{\text {likelihood }}^{j<K} \mathbf{M}(\mathcal{L}(j), j)\right) \times\left(P_{0}(\mathcal{L}(0)) \prod_{j=1}^{j<K-1} \mathbf{S}(\mathcal{L}(j), \mathcal{L}(j+1))\right)
$$

where $\widetilde{\mathcal{L}}$ is the solution. Equation 3 depicts a combinatorial optimization problem, since the number of possibilities for state constellations are finite. The evaluation of the cost function in this problem is computationally inexpensive, therefore standard optimization techniques such as simulated annealing could be used. However, since the problem can be looked at as overlapping sub-problems, we could use Dynamic Programming to find the optimal solution [2]. Similar to Dijkstra shortest path algorithm, we can define and store the maximum cost of getting at the time index $j$ in a $(L+1) \times K$ matrix $\mathbf{Q}$ :

$$
\mathbf{Q}(i, j)=\left\{\begin{array}{lc}
\infty & j<0 \\
\mathbf{M}(i, j) \times P_{0}(i) & j=0 \\
\mathbf{M}(i, j)+\max _{i}(\mathbf{Q}(i, j-1) \times \mathbf{S}(i, j)) & \text { otherwise }
\end{array}\right.
$$

Once the matrix $\mathbf{Q}$ in the equation 4 is computed recursively, the solution of the optimization problem in the equation 3 is:

$$
\widetilde{\mathcal{L}}(j)=\tilde{i}=\arg \max _{i} \mathbf{Q}(i, j) \quad \text { for } \quad j \in[0, K] .
$$

Estimated mapping function $\widetilde{\mathcal{L}}$ can be used in equation 2 to compute the updated gating surrogate interval.

\section{Experimental Results}

To evaluate the performance of the proposed method, we define two error measures. First error metric $e_{1}$ is the percentage of wrongly labeled fluoroscopic frames. Second error measure $e_{2}$ is the percentage of wrongly labeled "no-match". Although the wrongly labeled "no match" does not have any affect on the computed gating surrogate interval, it has an adverse impact on the duty cycle.

For the first series of tests, we used a breathing phantom that has a moving target within a hallow cavity. The speed and range of the target motion within the phantom can be adjusted. A ten phase 4DCT of the breathing phantom was acquired using Siemens Sensation 64 CT Scanner (Siemens Medical Solutions, Med CT, Forchheim, Germany). Simulator fluoroscopic sequences of the phantom were taken using Siemens Mevasim S Simulator (Siemens Medical Solutions, Med OCS, Concord CA, USA) from the phantom with three different motion ranges and speeds. For the second and third series, the 4DCT of patients with synthetic and real fluoroscopic sequences were used. We acquired two 4DCT 


\begin{tabular}{lcc} 
Test & $e_{1}(\%)$ & $e_{2}(\%)$ \\
\hline Phantom Original (i.e., same as 4DCT) & $0 \%$ & $0 \%$ \\
Phantom Slow Breathing & $8 \%$ & $0 \%$ \\
Phantom Fast Breathing & $5 \%$ & $0 \%$ \\
Phantom Deep Breathing & $8 \%$ & $15 \%$ \\
Phantom Shallow Breathing & $8 \%$ & $15 \%$ \\
\hline Patient1 with Syn. Fluo. & $4 \%$ & $10 \%$ \\
Patient1 with Syn. Fluo. Modified Pattern 1 & $7 \%$ & $10 \%$ \\
Patient1 with Syn. Fluo. Modified Pattern 2 & $8 \%$ & $10 \%$ \\
\hline Patient2 with Fluo. & $15 \%$ & $15 \%$ \\
\hline Patient3 with Fluo. & $14 \%$ & $10 \%$ \\
\hline Average & $7 \%$ & $8.5 \%$
\end{tabular}

Table 1. Results of the two error measures for various datasets.

scans of the same patient at two different time points. We generated DRRs from one the 4DCT phases and puzzled them together to form synthetic fluoroscopic sequences. We generated three sequences. For the first, we concatenate the original $4 \mathrm{DCT}$ phases multiple times. For the second and third, we varied the sequence order and generated two distinct synthetic breathing patterns.

Finally, we acquired two 4DCT scans of the two different patients with the corresponding simulator acquisitions. In all the cases, correct correspondences were manually selected. We used split-screen and a blending display method to identify the correct correspondences for each fluoroscopic frame out of various $4 \mathrm{DCT}$ phases. Sample images and the similarity matrix with the overlaid mapping function are depicted in Figure 3 . The results of the two error measures are brought in the table 3 . The processing time depends on the number of fluoroscopic sequence frame and it is roughly one second per frame. All the tests were performed on an Intel Centrino Duo CPU with $2.0 \mathrm{GHz}$ and $2 \mathrm{~GB}$ of RAM and a NVIDIA Quadro FX 2500 display adapter.

\section{Summary and Conclusion}

We have developed an image based mapping/synchronization procedure that automatically labels pre-treatment fluoroscopic image frames with the corresponding phase from 4DCT. The mapping procedure is formulated as an optimization process, which finds an optimal mapping maximizing the image similarity between the corresponding pairs, while preserving a temporal coherency to an established Markov model for breathing. The mapping procedure also detects the frames with no corresponding phase from the planning 4DCT. The fluoroscopic image based verification addresses the problem of establishing the correlation between surrogate signal and internal target prior to treatment. By virtue of having the labels generated from a mapping process, we can adaptively change the surrogate gating interval to the up-to-date breathing pattern of the patient. Results on phantom, synthetic, and patient data shows in average 93 percent of frames are correctly labeled. 

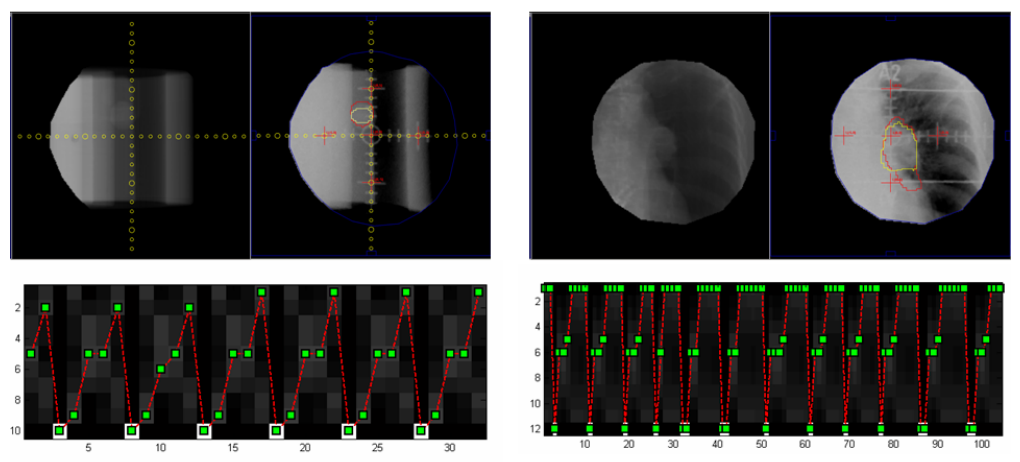

(a)

(b)

Fig. 3. (a) and (b) depicts a 4DCT frame with matching fluoroscopic frame with overlaid contours for phantom and patient datasets, respectively. The bottom row shows the corresponding image similarity matrix for each case.

\section{References}

1. Baum, L. E. ,Petrie T., Soules G. , and Weiss N., A maximization technique occurring in the statistical analysis of probabilistic functions of Markov chains, Ann. Math. Statist., 1970, 41(1) : pp. 164-171.

2. Bellman, R., Dynamic Programming, Princeton Univ. Press, Princeton, NJ, 1957.

3. Berbeco, R.I., et. al., Towards fluoroscopic respiratory gating for lung tumours without radiopaque markers. Phys Med Biol, 2005, 50(19): p. 4481-90.

4. Huanmei, W., Sharp, G. C., et. al., A finite state model for respiratory image guided radiation therapy, Phys. Med. Biol. 2004, 49, pp 5357-5372.

5. -

6. Keall, P.J., V.R. Kini, S.S. Vedam, and R. Mohan, Motion adaptive x-ray therapy: a feasibility study. Phys Med Biol, 2001, 46(1): pp 1-10.

7. Keall P. J., et. al., The management of respiratory motion in radiation oncology report of AAPM Task Group 76, Med Phys., 2006, 33(10): pp 3874-900.

8. Kubo, H.D. and B.C. Hill, Respiration gated radiotherapy treatment: a technical study. Phys Med Biol, 1996, 41(1): pp 83-91.

9. Lawrence R. Rabiner, A tutorial on Hidden Markov Models and selected applications in speech recognition, Proceedings of the IEEE, 1989, 77 (2): pp 257-286.

10. Onimaru, R., et. al., The effect of tumor location and respiratory function on tumor movement estimated by real-time tracking radiotherapy (RTRT) system. Int J Radiat Oncol Biol Phys, 2005, 63(1): pp 164-9.

11. Schweikard, A., et. al., Robotic motion compensation for respiratory movement during radiosurgery. Comput Aided Surg, 2000. 5(4): pp. 263-77.

12. Shirato, H., et. al., Feasibility of insertion/implantation of 2.0-mm-diameter gold internal fiducial markers for precise setup and real-time tumor tracking in radiotherapy. Int J Radiat Oncol Biol Phys, 2003, 56(1), pp 240-7.

13. Rangaraj, D. and L. Papiez, Synchronized delivery of DMLC intensity modulated radiation therapy for stationary and moving targets. Med Phys, 2005, 32(6): pp 1802-17. 
14. Rietzel, E., Chen, G. T. T., Improving retrospective sorting of $4 \mathrm{D}$ computed tomography data, Med Phys, 2006, 33(2): pp 377-379. 